\title{
An Advanced Technique for Volumetric Analysis
}

\author{
R. B. Dubey \\ Apeejay College of \\ Engg. \\ ICE Deptt.Sohna, \\ Gurgaon, India.
}

\author{
M.Hanmandlu, \\ Sr. Member IEEE \\ Electrical Engg. Deptt. \\ IIT, New Delhi, India.
}

\author{
S. K. Gupta \\ Vaish College of \\ Engg. \\ Rohtak, India.
}

\author{
S. K. Gupta \\ Electrical Engg. \\ Deptt. \\ DCRUST,Murthal, \\ Sonepat, India.
}

\begin{abstract}
:
An accurate segmentation is critical, especially when the tumor morphological changes remain subtle, irregular and difficult to assess by clinical examination. This quantitative measurement depends on the accuracy of the segmentation method used. The undesired partial volume effect, which lies on a boundary between a high intensity region and low intensity region, makes unerring boundary determination a difficult task. A new approach to segmentation is proposed that removes the adverse effect on the boundary, which is unwanted especially from the point of view of volume rendering. This approach gives more accurate boundary detection and holes filling after segmentation. A semi-automatic calculation of volumetric size of brain tumor has been implemented in this approach. A comparative analysis of manual, seeded region growing and this advance approach shows more accurate and better performance for $3 \mathrm{D}$ volume measurements. This method is tested by two patients of different tumor type and shape, and better results are reported.
\end{abstract}

\section{Key words:}

Brain tumor segmentation, MRI, seeded region-growing segmentation and advanced gradient magnitude region growing technique.

\section{INTRODUCTION}

Brain tissue and tumor segmentation in MR images has been an active area of research today [1-3]. In general the problem of image segmentation involves clustering of similar feature vectors [4-5]. Extraction of good features is thus fundamental to successful image segmentation. The segmentation task becomes more challenging when one wants to derive common decision boundaries on

different object types in a set of images. Due to the complex structure of different tissues such as white matter (WM), gray matter (GM) and cerebrospinal fluid (CSF) in the brain images, extraction of useful feature is a fundamental task. Intensity is an important feature in discriminating different tissue types in brain MR images. However, using intensity feature alone to segment complex brain tissue and tumor in a single modality MR image has been proved to be insufficient [2-8].

The MR imaging method is the best due to its higher resolution than the other methods. Its resolution is approximately 100 microns [9-10]. MR imaging is currently the method of choice for early detection of brain tumor in human brain. However, the interpretation of MRI is largely based on radiologist's opinion. Generalization of brain screening programs requires efficient double reading of MR image, which allows reduction of false negative interpretations, but it may be difficult to achieve. Computer aided detection systems are dramatically improving and can now assist in the detection of suspicious brain lesions, suspicious masses. The task of manually segmenting brain tumors from MR imaging is generally time consuming and difficult. An automated segmentation method is desirable because it reduces the load on the operator and generates satisfactory results [11].

The region growing segmentation is used to segment the brain tumors due to its wide range of applications and automatic features. After taking the image of the tumorous brain there is a need to process it. The image clearly shows the place of the tumorous portion of the brain. The image does not give the information about the numerical parameters such as area and volume of the tumorous portion of the brain. After segmentation the desired tumor area is selected from the segmented image. This selected region is used to calculate the area and volume of the tumor present in the MR image [10-16, 20].

Manual volumetric method is gold standard approach for 3D quantitative measurements. The main disadvantage of this method is that it is labor intensive and time consuming. Segmentation of region of interest in volumetric medical images is still a challenging problem. Current survey proves that region growing is an effective approach for image segmentation especially for the homogenous regions. The disadvantage of region growing is the partial volume effect [45]. The partial volume effect limits the accuracy of MRI brain image segmentation. It blurs the intensity distinction between tissues classes at the border of the two tissues types because voxel may represent more than one kind of tissue types. M. Sato et al., developed a suitable modification in region growing technique. This modification is called advanced gradient magnitude region growing technique (AGMRGT) used to remove the partial volume effects and to incorporate gradient information for more accurate boundary detection and filling holes occurred after segmentation [45-46].

The remainder of this paper is organized as follows. Section II reviews the relevant previous literature and highlights the research motivation. Section-III presents materials and methods of the problem. Section-IV discusses results and Section-V draws the conclusions.

\section{LITERATURE REVIEW}

The segmentation task can be region-based or edge-based. However majority of MRI-based segmentation methods in the literature are region-based. The recent methods involving deformable models also come under the edge-based category. In the case of MRI segmentation, uncertainty is introduced due to factors such as partial volume effects, integration of multi- 
protocol image data and observer variability. Most of the segmentation methods appear in the statistical framework, providing a classification of the image data into different tissue types, while there exist only a few using the deterministic approach.

\section{Deterministic Approaches}

Gibbs et al. [18] introduced a morphological edge detection technique combined with simple region growing to segment enhancing tumors on T1 MRI data. Based on an initial sample of the enhanced tumor signal and the surrounding tissues, provided manually, an initial segmentation is performed combining pixel thresholding, fitting to an edge map of the image data and morphological opening and closing, inspired by the work proposed by Kennedy et al. [19]. The tumor area is defined based on pixel values in the range of 4 standard deviations around the mean value, constrained by the edge map. Letteboer et al. [20], proposed an interactive segmentation method for three types of tumors: full enhancing, ring enhancing and non-enhancing. After manual tracing of an initial slice, a series of morphological filtering operations based on the watershed algorithm is applied to partition the MRI volume data into homogeneous areas. A multiscale framework is employed to correlate segmented regions across different scales. Droske et al. [21], proposed to use a deformable model, implemented with a level set formulation, to partition the MRI data into regions with similar image properties, based on prior intensity-based pixel likelihoods for tumor tissues. The deformable model optimization is performed on a spatially adaptive grid, only refined in inhomogeneous regions. Homogeneity measures included gray value intervals, defined from a user input and image gradient values. Some manual supervision of the deformable model is required, so that incremental segmented areas are proposed to the user who controlled the final segmentation results.

\section{Statistical Approaches}

Vaiddynathan et al. [22] compared two supervised multispectral classification methods: $\mathrm{k}$ nearest neighbour $(\mathrm{kNN})$ and spectral fuzzy C-means (FCM). For these two classification approaches, nine tissue classes are considered (background, CSF, WM, GM, fat, muscle, tumor, edema and necrosis). The authors also tested an interactive seed-growing segmentation approach on T1E MRI data. The seed-growing algorithm only segmented tumor tissue based on a sample pixel population manually selected by the user. Clark et al. [23] introduced a knowledge-based (KB) automated segmentation method for glioblastomas on multispectral data combining T1E, PD and T2 weighted data. A training phase is performed on 17 slices from seven patients, extracting tumor size and enhancement level characteristics. Slices are first characterized as normal or abnormal via a fuzzy C-means (FCM) classification and the analysis is of the clustering result through an expert system. A final processing stage is performed, based on histogram analysis of the tumor pixels and heuristics on the density of intensity features of non-tumor tissues. Indeed, based on the observation that tumors can show different levels of enhancement and very complex shapes, the final $\mathrm{KB}$ approach is focused on characterizing non-

tumoral tissues. Kaus et al. [24] presented a complete validation of an automated segmentation method on T1E data from twenty patients with meningiomas and low-grade gliomas. The segmentation method, called an adaptive template-moderated classification and described in [25-26] is based on an iterative process. It alternated between a kNN classification of voxels into five hierarchical tissue types (background, skin-fat-bone, brain, ventricles and tumor) and a nonlinear registration of the data with an anatomical atlas to align the data with the template. The kNN classification used features from data intensity values and anatomical priors on the tissue location from the atlas. This method performed extraction of the five tissues in a predetermined hierarchical order. Tissue mean values are learned on the patients data via manual selection of three or four points for each tissue. To handle the presence of the tumor in the registration process, voxels assigned to the tumor class are masked with brain labels prior to registration with the atlas. This method obviously relied on a strong homogeneity assumption of the tumors appearance on MRI data, which is reinforced by the use of anisotropic diffusion filtering.

Moonis et al. [27] proposed a segmentation framework based on fuzzy connectedness (FC), which optimally clustered voxels into classes of high connectivity. The method is applied to T1, T1E and T2 data and initialized with an MRI data standardization of the gray levels based on non-linear transformation of the histograms [28]. Liu et al. [29], from the same group, used a similar approach based on a volume of interest on co-registered $\mathrm{T} 1$ and $\mathrm{T} 2$ data, to process only slices containing the tumor. A set of points inside the tumor is selected to initialize the statistics used in the FC. The threshold level applied to the FC maps to define the final segmentation result is determined empirically on five datasets and then fixed once for all. Segmentation is performed separately on the T2, T1E and subtracted (T1-T1E) data sets in 3D. Experts perform manual corrections of the segmentation results. Fletcher-Heath et al. [30], proposed a combination of unsupervised classification with FCM and knowledge-based (KB) image processing for segmentation of non-enhancing tumors. The FCM is run on spectral data (T1, T2 and PD). As the authors pointed out, FCM tended to define clusters with similar sizes, which required an initial classification in ten classes. A KB system is then designed to re-cluster the segmentation results into seven classes based on a training phase. Difficulties principally arose in the separation of CSF and tumor signals. Mazzara et al. [31-32], compared the kNN approach from and the KG-based approach from [22-23] for growth tumor volume (GTV) measurements on eleven patients with high and low-grade gliomas. As used in oncology radiation therapy, GTV corresponded to the area enclosing several contiguous clusters of enhancing pixels. The study showed severe limitations of the KGsystem in handling particular cases such as non-enhancing tumor margins or the presence of non-enhancing cystic necrotic tissues at the center of the tumor. On the other hand, the $\mathrm{kNN}$ segmentation method, trained with sample data from MRI slices to segment, lead to robust segmentation results on all patients. Beyer et al. [32], from the same group, presented a similar and more recent comparative study, extracting GTV with the same two segmentation methods and evaluating the results in terms of predictive dose measurement for therapy planning.

Zou et al. [33], proposed a continuous probabilistic segmentation framework, based on mixture modeling for two classes: tumor and non-tumor tissues. After initialization of the segmentation with the semi-automated method from Kaus et al. [24], the segmentation process involved estimation of the distribution parameters and probability values thresholding. Three 
metrics are proposed and evaluated to optimize the threshold selection: Receiver operating curve (ROC), which weights the sensitivity versus the specificity of the segmentation result, a dice similarity coefficient, which is also a function of sensitivity and specificity and mutual information that directly compares the segmentation result to a ground truth. Prastawa et al. [34] proposed a segmentation framework based on outlier detection on T2 data. The abnormal tumor region is detected via registration on a normal brain atlas. Statistical clustering of the abnormal voxels, followed by a deformable model, is then used to isolate the tumor and the edema.

A technique that enables automatic accurate (54\%) and robust segmentation and separation the pelvis and the femur, more specifically, of the acetabulum and the femoral head in the hip joint from 3-D CT images is presented by Zoroofi et al [35]. Vertebral extraction from digital videofluoroscopic (DVF) images has been a popular goal for a considerable time. However, this has been proved to be very difficult due to the poor image quality. Vertebral extraction is an image-processing problem and consequently, algorithms from the field of computer vision could have a valuable role to play. The Hough transform (HT) has many desirable properties, especially good resistance against noise and might be suitable for coping with the problems inherent with DVF images. Y. Zheng et al. [36] applied it to vivo DVF lumbar spine images and gave promising results. The great advantage of the HT is that it can locate the same vertebral contour in the sequences and thus, contour shape will not change, which is important when the vertebral image suffers noise or occlusion. This paper only shows the changing pattern of the lumbar spine vertebral centers. This can provide clinicians with valuable information for diagnosis of spinal disorders. A refined segmentation-by-registration scheme is propose in which an atlas based segmentation of the pathological lungs is refined by applying voxel classification to the border volume of the transformed probabilistic atlas. It is shown that this refinement step introduces a significant improvement in segmentation accuracy compared to a standard segmentation-by-registration approach. No statistical difference in performance is found between voxel classification and the registration method. The registration however gives more visually pleasing results than the voxel classification due to its implicit lung model [37].

Y. Zia et al. [38] presented an automated algorithm driven by MR data and anatomic knowledge to extract the caudate nucleus $(\mathrm{CN})$, which has a simple shape but lacks clearly defined MRI intensity boundaries, from human MR brain images. A high level of consistency between results generated by the algorithm and those by expert tracing is demonstrated for $\mathrm{CN}$ volume and spatial characteristics. The algorithm presented here has several advantages for the neuroimaging researcher. First, it is highly automated, only requiring the user to designate the locations of the anterior and posterior commissures (AC and PC) as initial input. No manual supervision or intervention is required after this initial input step. Second, it is rapid and efficient, extracting the $\mathrm{CN}$ nucleus within half a minute, while expert delineation takes on average 30 minutes. The method mainly includes three steps: structure element subdivision, feature extraction, feature selection and classification. Experimental results demonstrate the feature selected by this method can contribute effective and complementary information to discriminating tumor and normal tissues. The selected features include intensity, symmetry and texture based features extracted from multi-protocol MR images. By the comparison with $\mathrm{kNN}$ and support vector machine (SVM) methods, it shows that feature selection reduces the dimensionality of the feature space and improves the performance of the classifier. The proposed method can perform better than the existing segmentation methods such as active contour model (ACM) and fuzzy connectedness based method and achieves very accurate segmentation results. On the basis of the available tumor segmentation results from MRI data it is extremely difficult to conclude which one is the best method. [39-42].

\section{MATERIALS AND METHODS}

Implementation of this novel approach contains various processing steps, like raw image collection, image preprocessing, thresholding, image segmentation and volume calculation. The sequences of the processing steps are arranged in a meaningful manner as shown in Fig.1.

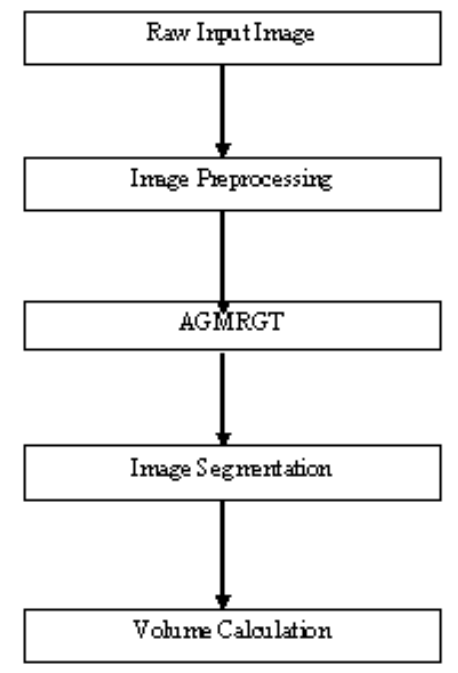

Fig.1: The Proposed methodology.

3.1. MRI Data: The MR images are acquired on a $1.5 \mathrm{~T}$ using T2-weighted axial contrast images. The other specifications of image are: thickness of image slice- $0.5 \mathrm{~cm}$, number of images per data set -23 and image format- Gif.

3.2. Preprocessing: Image preprocessing is the very first step in the image processing. In this process the image is converted into the accessible form. Also image 'intensity adjustment' and 'noise reduction' processes are carried out. After this the Gaussian image filtering is performed. Filtering is necessary because it reduces the noise present in the image.

\subsection{Seeded region growing}

Region growing group's pixels or sub regions into larger regions based on predefined criteria. The basic idea is to start with a pixel or a group of pixels and examine the neighboring pixels. If a neighboring pixel meets a certain criteria, it is added to the group and if it does not meet the criteria, it is not added. This process is continued until no more neighboring pixels can be added to the group. Thus, a region is defined. In this method, the point is to group pixels of the same or similar brightness or color into region according to the given membership criteria. The membership criteria are based on some threshold value. The membership in a region can be based on multiple criteria. 
The confidence connected region-growing algorithm based on extraction of connected set of pixels whose pixel intensities are consistent with the pixel of a seed point. First, the mean and variance across an 8-connected neighborhood are calculated for a seed point. The pixels connected to this seed point whose values are within the confidence interval for the seed point are grouped. The width of this confidence interval is controlled by the user defied factor $f$ according to equation (1)

$$
I(x) \in[m-f \sigma, m+f \sigma]
$$

Where $m$ and $\sigma$ are the mean and standard deviation of the region intensities, $\mathrm{f}$ is a factor defined by the user. Small values of the factor $f$ will restrict the inclusion of pixels to those having very similar intensities to those in the current region. Larger values of this multiplier factor will relax the accepting condition and will result in more generous growth of the region. $I$ is the image and $x$ is the position of the particular neighbor pixel being considered for inclusion in the region [43-44].

\subsection{Advanced gradient magnitude region growing technique (AGMRGT)}

In the first step proper threshold is chosen in order to distinguish the interior area from other organs in the whole volumetric image dataset. Then advanced gradient magnitude region growing algorithm is applied, in which gradient magnitude is computed by Sobel operator and employed as the definition of homogeneity criterion. This implementation allowed stable boundary detection when the gradient suffers from intersection variations and gaps. By analyzing the gradient magnitude, the sufficient contrast present on the boundary region that increases the accuracy of segmentation [45].

To calculate the size of segmented tumor the relabeled method based on remaps the labels associated with object in a segmented image such that the label numbers are consecutive with no gaps between the label numbers used. Any object can be extracted from the relabeled output using a binary threshold. Here, the algorithm is adjusted to extract and relabeled the tumor and then find its size in pixels. The algorithm works well in two stages.
The first stage is to determine the input image labels and the number of pixels in each label. The second stage is to determine the output requested region to get total number of pixels accessed. From the whole slices we can calculate the number of pixels into the 3D volume. Segmented areas are automatically calculated and multiplied by the MRI slice thickness plus the interslice gap to calculate a per- slice tumor volume. The total tumor volume is obtained by summing the tumor bearing slices [45-46].

\section{RESULTS}

The developed algorithm is used to know about the location and size of the tumor for volume calculation. It takes one image at a time for processing and shows the output

\begin{tabular}{|l|l|l|l|}
\hline $\begin{array}{l}\text { S. } \\
\text { No. }\end{array}$ & $\begin{array}{l}\text { Data } \\
\text { Set }\end{array}$ & $\begin{array}{l}\text { Tumor } \\
\text { Area } \\
\left(\mathrm{cm}^{2}\right)\end{array}$ & $\begin{array}{l}\text { Tumor } \\
\text { volume } \\
\left(\mathrm{cm}^{3}\right)\end{array}$ \\
\hline 1 & 1 & 1.1746 & 0.5872 \\
\hline 2 & 2 & 2.1858 & 1.0929 \\
\hline
\end{tabular}

Table 1: Measurement of volume using AGMRGT image at every step. The data set 1 has tumor

in the three images while the data set 2 has five tumorous images. These tumorous images are clearly visible in the dataset 1 and 2 . When algorithm executes, the results of processing for raw input images of data set 1 and 2 are shown in Fig.2 and Fig.3 respectively. Volumetric calculation for brain tumor using the different calculation methods and the relative errors for each method compared with manual segmentation method is tabulated in Table 2 .

The Relative Error (RE) for tumor volume can be calculated as $\operatorname{RE}(\%)=\left(\left|P-P^{\prime} / P^{\prime}\right|\right) \times 100$

Where $\mathrm{P}$ tumor volume using $3 \mathrm{D}$ semi-automatic region growing method, $\mathrm{P}^{\prime}$ is tumor volume calculated using manual method.

\begin{tabular}{|l|l|l|l|l|l|}
\hline Dataset & $\begin{array}{l}\text { AGMRGT } \\
\text { Tumor } \\
\text { Volume }\left(\mathrm{cm}^{3}\right)\end{array}$ & $\begin{array}{l}\text { Seeded } \\
\text { region } \\
\text { growing } \\
(\mathrm{SRG}) \\
\text { Tumor } \\
\text { Volume } \\
\left(\mathrm{cm}^{3}\right)\end{array}$ & $\begin{array}{l}\text { Manual } \\
\text { Volume } \\
\left(\mathrm{cm}^{3}\right)\end{array}$ & $\begin{array}{l}\text { Relative } \\
\text { Error }(\%) \\
(\text { AGMRGT) }\end{array}$ & $\begin{array}{l}\text { Relative } \\
\text { Error }(\%) \\
(\mathrm{SRG})\end{array}$ \\
& $\begin{array}{l}0.4330 \\
\text { First }\end{array}$ & 0.9753 & 0.6320 & $7.08 \%$ & $31.5 \%$ \\
Second & 1.0929 & 0.972 & 1.1234 & $1.80 \%$ & $14.07 \%$ \\
\hline
\end{tabular}

Table 2: Comparison of seeded region growing, semi-automatic AGMRGT with manual segmentation technique. 

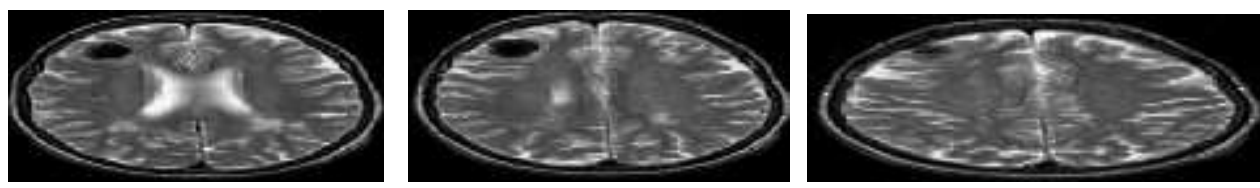

Fig. 2 (a): Original input MR image of dataset 1.
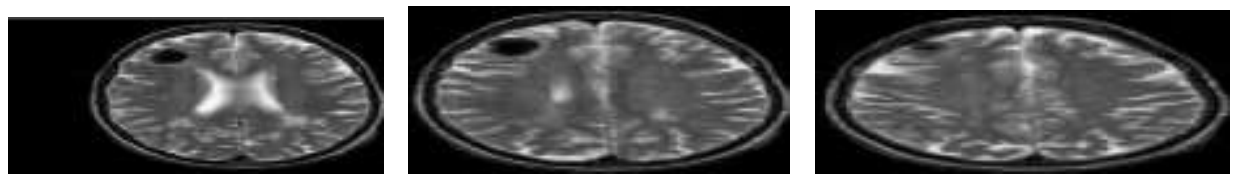

Fig.2 (b): Gaussian filtered image of dataset 1.
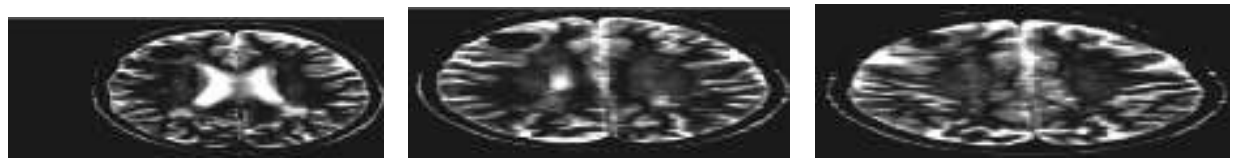

Fig. 2(c): Intensity adjusted image of dataset 1.

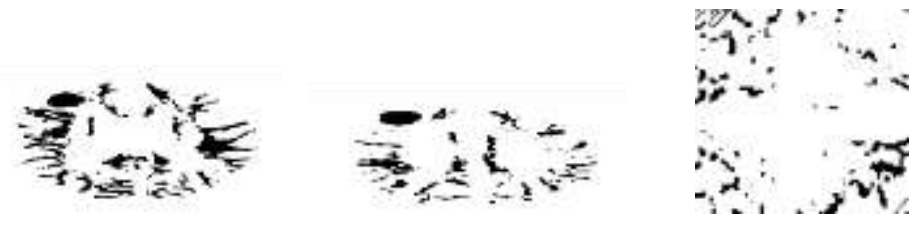

Fig. 2(d): Segmented image of dataset 1.
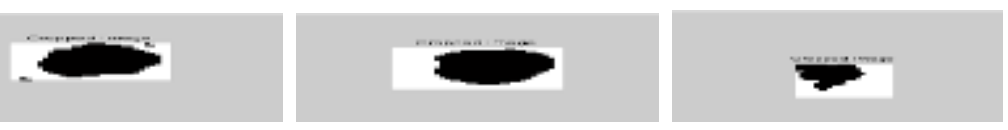

Fig.2 (e): Extracted tumor of dataset 1.

Fig. 2: The results after segmentation for data set 1.
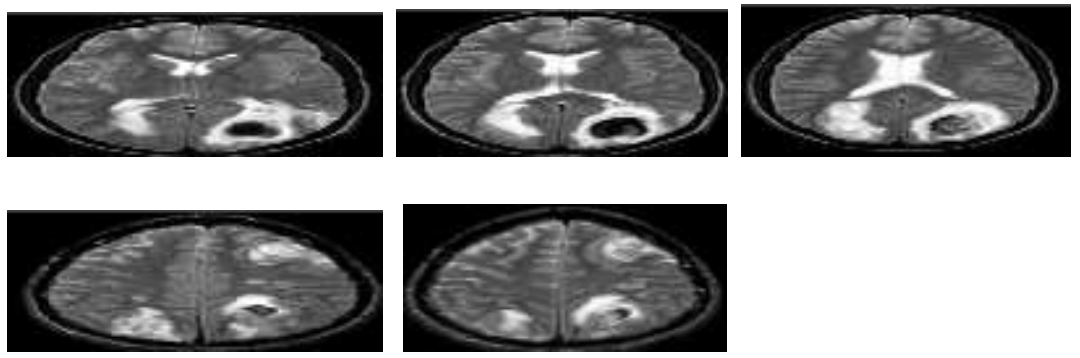

Fig. 3 (a): Original input MR image of dataset 2. 



Fig. 3 (b): Gaussian filtered image of dataset 2.
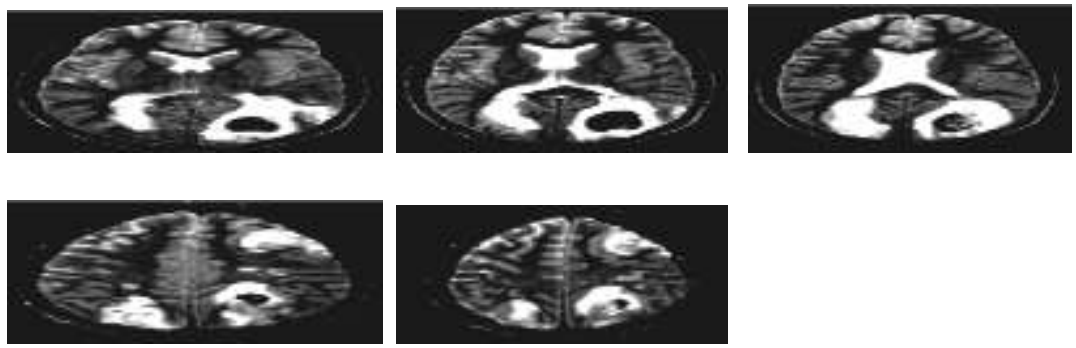

Fig. 3 (c): Intensity adjusted image of dataset 2.

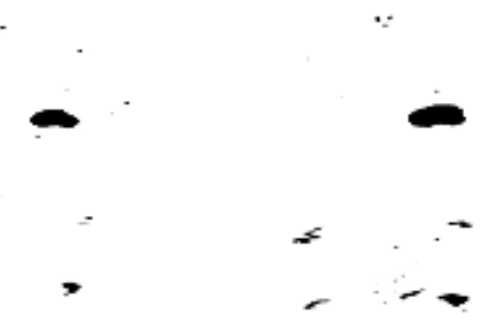

Fig. 3 (d): Segmented image of dataset 2.
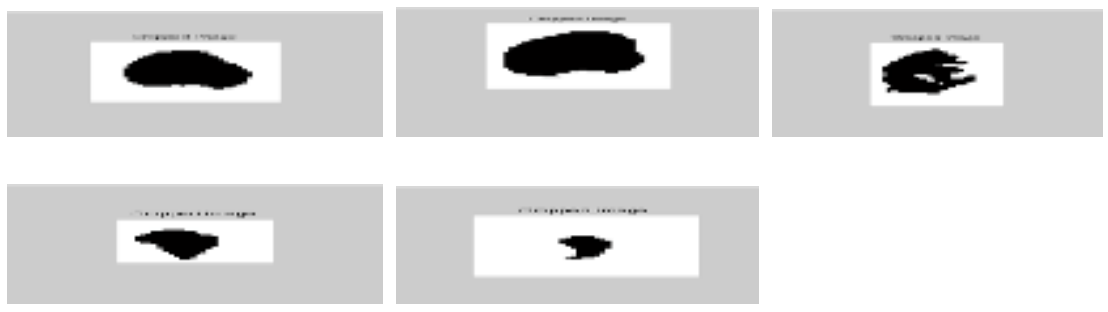

Fig. 3 (e): Extracted tumor of dataset 2.

Fig. 3: The results after segmentation for data set 2.

\section{CONCLUSIONS}

Imaging plays a central role in the diagnosis and treatment planning of brain tumor. Tumor volume is an important diagnostic indicator in treatment planning and results assessment for brain tumor. The measurement of brain tumor volume can assist tumor staging for

effective treatment surgical planning. The measurement of tumor volume using manual method is although gold standards approach but tedious, labor intensive and time consuming. It involves the tracing the tumor outline and tumor volume is derived by summation of total slices area. Whether this process is done by a radiologists or by a technologist, there is always and important element of subjectivity that results in both intra and inter-operator performance. In this study, a semi-automated AGMRGT system 
for brain tumor volume measurements is developed which overcome the problem of inter-operator variance, besides partial volume effects and shows satisfactory performance for segmentation. This method is applied to 8-tumor contained MRI slices from 2 brain tumor patients' data sets of different tumor type and shape, and better segmentation results are achieved.

The attention has been given to improve the accuracy of segmentation by implementing essential modification in the region growing algorithm as advanced gradient magnitude region growing technique (AGMRGT). The Sobel gradient magnitude based region growing algorithm eliminates the partial volume effect problem on the boundary. In AGMRGT algorithm, a gradient magnitude is applied to its homogeneity criterion. This algorithm seriously considers the gradient of the boundary and its neighborhood for partial volume effect and allows us to conduct accurate segmentation based on gradient magnitude in addition to the intensity value. These modifications overcome the partial volume effect artifacts. Hence, applying AGMRGT to segment brain tumors has increased the accuracy of the volumetric measurements. The comparisons of volume measurement by manual, seeded region growing and AGMRGT ensures that tumor accuracy have been improved using AGMRGT. The results show that volume measurements obtained using AGMRGT method is in good agreement with manually segmented data. Further work is in progress to test larger set of tumor datasets to improve the accuracy of segmentation.

\section{REFERENCES}

[1] K. M. Iftekharuddin, "On techniques in fractal analysis and their applications in brian," Medical imaging systems: technology and applications, Analysis and Computational Methods, vol. 1, World Scientific Publications, ISBN 981256-993-6, 2005.

[2] L. P. Clarke, R. P. Velthuizen, M. A. Camacho, J. J. Heine, M. Vaidyanathan, L. O. Hall, R. W. Thatcher and M. L. Silbiger, "MRI segmentation: methods and applications", Magn. Reson. Imaging, vol. 13, Issue 3, pp. 343-368, 1995.

[3] J. C. Bezdek, L. O. Hall and L P. Clarke, "Review of MR image segmentation techniques using pattern recognition", Med. Phys., vol. 20, Issue 4, pp. 1033-1048, 1993.

[4] H. S. Zadech and H. S. Windham, "Optimal linear transformation for MRI feature extraction", IEEE Trans. Med. Imaging, v ol. 15, pp. 749-767, 1996.

[5] H. S. Zadech and J.P. Windham, "A comparative analysis of several transformations for enhancement and segmentation of magnetic resonance image scene sequences," IEEE Trans. Med. Imaging, vol. 11, no. 3, pp. 302-318, 1992.

[6] D. Wang and D. M. Doddrell, "A segmentation-based partial-volume-compensated method for an accurate measurement of lateral ventricular volumes on T1weighted magnetic resonance images," Magn. Reson. Imaging, vol. 19, pp. 267-272, 2001.

[7] M. Just and M. Thelen, "Tissue characterization with Tl, $T 2$ and proton density values: results in 160 patients with brain tumors," Radiology, vol. 169, pp. 779-785, 1988.

[8] K. M. Iftekharuddin, J. Zheng, M. A. Islam and R. J. Ogg, "Fractal-based brain tumor detection in multimodal MRI,"Journal of Applied Mathematics and Computation, pp. 1-19, 2008.
[9] S. M. Haney, P. M. Thompson, T. F. Cloughesy, J. R. Alger and A. W. Toga, "Tracking Tumor Growth Rates in

Patients with Malignant Gliomas: A Test of Two Algorithms," AJNR American Journal of Neuroradiology, vol. 22, pp. 73-82, January 2001.

[10] R. S. Khanpur,"Handbook of Bio-Medical Instrumentation", $2^{\text {nd }}$ edition 1999.

[11] K. Xie, J. Yang, Z. G. Zau and Y. M. Zhu," Semiautomatic Brain Tumor an edema segmentation using MRI", Europian Journal of Radiology, vol. 56 Issue 1, pp. 12-19, 2005.

[12] D. L. Pham, C. Xu and J. L. Prince, "Current Methods in Medical Image Segmentation”, Annual Review of Biomedical Engineering, volume 2, pp 315-337, 2000.

[13] R. Damadian, K. Zaner, D. Hor, T. DiMaio, L. Minkoff and M. Goldsmith, "Nuclear magnetic resonance as a new tool in cancer research: human tumors" by NMR, Ann N Y Acad Sci, vol. 222, pp.1048-76, 1973.

[14] M. Edman, "Segmentation Using a Region Growing Algorithm", Rensselaer Polytechnic Institute, October 18, 2007.

[15] http://en.wikipedia.org/wiki/Segmentation_(image processing)

[16] Y. Yang, X. Yan, C. Zheng and P. Lin, "A Novel Statistical Method for Segmentation of Brain MRI”, IEEE, pp. 946-949, 2004.

[17] J. Hwang, Y. Han and H. W. Park, "Segmentation of Brain Parenchyma using Bilateral Filtering and Region Growing”, International Conference of IEEE EMBS, pp. 6263-6266, 2007.

[18] P. Gibbs, DL. Buckley, SJ. Blackband, et al., "Tumour volume determination from MR images by morphological segmentation," Phys Med Biol, vol. 41, Issue 11, pp. 2437-46, 1996.

[19] DN. Kennedy, PA. Filipek and V. Caviness, "Anatomic segmentation and volumetric calculations in nuclear magnetic resonance image," IEEE Trans Med Imag, vol. 8, pp. 1-7, 1989.

[20] MMJ. Letteboer, OF. Olsen, EB. Dam, et al., "Segmentation of tumors in magnetic resonance brain images using an interactive multiscale watershed algorithm," Acad Radiol, vol. 11, pp. 1125-1138, 2004.

[21] M. Droske, B. Meyer, M. Rumpf, et al., "An adaptive level set method for interactive segmentation of in tracranial tumors", Neuro Res, vol. 27, no. 4, pp. 363-70, 2005.

[22] M. Vaidyanathan, LP. Clarke, R. P. Velthuizen, et al., "Comparison of supervised MRI segmentation methods for tumor volume determination during therapy," Magn Reson Imaging, vol. 13, no. 5, pp. 719-28, 1995.

[23] M. Clark, LO. Hall, DB. Goldgof, et al., "Automatic tumor segmentation using knowledge-based techniques,"IEEE Trans Med Imag, vol. 17, no. 2, pp. 187-201, 1998.

[24] MR. Kaus, SK. Warfield, A. Nabavi, et al., "Automated segmentation of MR images of brain tumors," Radiology, vol. 218, Issue 2, pp. 586-91, 2001.

[25] MR. Kaus, SK. Warfield, FA. Jolesz, et al., "Segmentation of meningiomas and low grade gliomas in MRI,'International Conference on Medical Image Computing and Computer Assisted Intervention., Cambridge, UK, 1999. 
[26] SK. Warfield, MR. Kaus, FA. Jolesz, et al., "Adaptive template moderated spatially varying statistical classification," International Conference on Medical Image Computing and Computer-Assisted Intervention (MICCAI) Cambridge, MA, USA, 1998.

[27] Moonis et al., "Estimation of Tumor Volume with Fuzzy Connectedness Segmentation of MR images", ATRN American Journal of Neuroradiology, vol. 23, pp. 356363, 2002.

[28] LG. Nyul and JK. Udupa, "On standardizing the MR image intensity scale,"Magn Reson Med, vol. 42, pp. 1072-1081, 1999.

[29] J. Liu, JK. Udupa,D. Odhner, et al., “A system for brain tumor volume estimation via MR imaging and fuzzy connectedness," Comput Med Imaging Graph, vol. 29, Issue 1, pp. 21-34, 2005.

[30] LM. Fletcher-Heath, LO. Hall, DB. Goldgof, et al., "Automatic segmentation of non-enhancing brain tumors in magnetic resonance images," Artif Intell Med, vol. 21, Issue (1-3), pp. 43-63, 2001.

[31] GP. Mazzara, RP. Velthuizen, JL. Pearlman, et al., “Brain tumor target volume determination for radiation treatment planning through automated MRI segmentation," Int J Radiat Oncol Biol Phys, vol. 59, Issue 1, pp. 300-12, 2004.

[32] GP.Beyer, RP. Velthuizen, FR. Murtagh, et al., "Technical aspects and evaluation methodology for the application of two automated brain MRI tumor segmentation methods in radiation therapy planning," Magn Reson Imaging, vol. 24, Issue 9, pp. 1167-78, 2006.

[33] KH. Zou, WM. Wells, R. Kikinis, et al., "Three validation metrics for automated probabilistic image segmentation of brain tumours, "Stat Med, vol. 23, Issue 8, pp.1259-82, 2004.

[34] M. Prastawa, E. Bullitt, S. Ho, et al,., "A brain tumor segmentation framework based on outlier detection, Med Image Anal, vol. 8, pp. 275-283, 2004.

[35] R. A. Zoroofi, Y. Sato, T. Sasama, T. Nishi, N. Sugano, K. Yonenobu, H. Yoshikawa, T. Ochi and S. Tamura, "Automated segmentation of acetabulum and femoral head from 3-D CT images", IEEE Transaction on Information Technology and Biomedicine, vol. 7, no. 4, pp329-343, 2003.

[36] Y. Zheng, M. S. Nixon and R. Allen, "Automated Segmentation of Lumbar Vertebrae in Digital Videofluoroscopic Images", IEEE Trans. on Medical Imaging, vol. 23, no. 1, pp. 45-52, 2004.
[37] I. Sluimer, M. Prokop and B. V. Ginneken, "Toward Automated Segmentation of the Pathological Lung in CT", IEEE Trans. on Med. Imaging, vol. 24, no. 8, pp. 1025-1038, 2005.

[38] Y. Xia, K. Bettinger, L. Shen and A. L. Reiss, "Automatic Segmentation of the Caudate Nucleus From Human Brain MR Images", IEEE Trans. on Med. Imaging, vol. 26, no. 4, pp. 509-517, 2007.

[39] X. Xuan and Q. Liao, "Statistical structure analysis in MRI brain tumor segmentation", IEEE Comp. society, International Conf. on Image And Graphics, pp. 421-426, 2007.

[40] E. Angelini, J. Atif, J. Delon, et al., "Detection of glioma evolution on longitudinal MRI studies,"International Symposium on Biomedical Imaging (ISBI), Arlington USA, 2007.

[41] H. Khotanlou, O. Colliot, J. Atif, et al., "3D brain tumor segmentation in MRI using fuzzy classification, symmetry analysis and spatially constrained deformable models," Fuzzy Sets and Systems, 2007.

[42] W. Dou, S. Ruan, Y. Chen, et al., "A framework of fuzzy information fusion for segmentation of brain tumor tissues on $R$ images," Image and Vision Computing, vol. 25, pp. 164-172, 2007.

[43] C. Rafael, Gonzalez and E. Richard Woods, "Digital Image Processing", $2^{\text {nd }}$ edition, 2003.

[44] Y. Lu, T. Jiang and Y. Zang, "A Split-Merge-Based Region Growing Method for fMRI Activation Detection," International Journal of Human Brain Mapping: WileyLiss, Inc., pp. 1-9, 2004.

[45] M. Sato, S. Lakare, M. Van and A. Kaufaman, "A gradient magnitude based region growing algorithm for accurate segmentation", Proceeding of international conference on image processing, vol. 3, pp. 448-451, 2000.

[46] S. Saraswathy, F. Crawford and S. J. Nelson, "Semiautomated segmentation of brain tumor lesions in $M R$ images," Proc. Intl. soc. Mag. Reson. Med., vol. 14, pp. 1609, 2009. 